D. Blackmore ${ }^{\mathrm{a}}$, A. Prykarpatski ${ }^{\mathrm{b}}$, M. Vovk $^{\mathrm{c}}$, P. Pukach ${ }^{\mathrm{d}}$, Ya. Prykarpatsky $^{\mathrm{e}}$

\title{
THE PFEIFFER-LAX-SATO TYPE VECTOR FIELD EQUATIONS AND THE RELATED INTEGRABLE VERSAL DEFORMATIONS
}

\begin{abstract}
D. Blackmore, A. Prykarpatski, M. Vovk, P. Pukach, Ya. Prykarpatsky. The Pfeiffer-Lax-Sato type vector field equations and the related integrable versal deformations, Mat. Stud. 48 (2017), 124-133.

We study versal deformatiions of the Pfeiffer-Lax-Sato type vector field equations, related with a centrally extended metrized Lie algebra as the direct sum of vector fields and differential forms on torus.
\end{abstract}

1. The Pfeiffer-Lax-Sato vector field equations. Consider for simplicity a vector field $X: \mathbb{R} \times \mathbb{T}^{n} \rightarrow T\left(\mathbb{R} \times \mathbb{T}^{n}\right)$ on the $(n+1)$-dimensional toroidal cylinder $\mathbb{R} \times \mathbb{T}^{n}$ for arbitrary $n \in \mathbb{Z}_{+}$, which we will write in the slightly special form

$$
A=\frac{\partial}{\partial t}+\left\langle a(t, x), \frac{\partial}{\partial x}\right\rangle
$$

where $(t, x) \in \mathbb{R} \times \mathbb{T}^{n}, a(t, x) \in \mathbb{E}^{n}, \frac{\partial}{\partial x}:=\left(\frac{\partial}{\partial x_{1}}, \frac{\partial}{\partial x_{2}}, \ldots, \frac{\partial}{\partial x_{n}}\right)^{\top}$ and $\langle\cdot, \cdot\rangle$ is the standard scalar product on the Euclidean space $\mathbb{E}^{n}$. With the vector field (1) one can associate the linear equation

$$
A \psi=0
$$

for some function $\psi \in C^{2}\left(\mathbb{R} \times \mathbb{T}^{n} ; \mathbb{R}\right)$, which we will call an "invariant" of the vector field.

Next, we study the existence and number of such functionally-independent invariants to the equation (2). For this let us pose the following Cauchy problem for equation (2): Find a function $\psi \in C^{2}\left(\mathbb{R} \times \mathbb{T}^{n} ; \mathbb{R}\right)$, which at point $t^{(0)} \in \mathbb{R}$ satisfies the condition $\left.\psi(t, x)\right|_{t=t^{(0)}}=$ $\psi^{(0)}(x), x \in \mathbb{T}^{n}$, for a given function $\psi^{(0)} \in C^{2}\left(\mathbb{T}^{n} ; \mathbb{R}\right)$. For the equation (2) there is a naturally related parametric vector field on the torus $\mathbb{T}^{n}$ in the form of the ordinary vector differential equation

$$
d x / d t=a(t, x),
$$

to which there corresponds the following Cauchy problem: find a function $x: \mathbb{R} \rightarrow \mathbb{T}^{n}$ satisfying

$$
\left.x(t)\right|_{t=t^{(0)}}=z
$$

2010 Mathematics Subject Classification: 17B68, 17B80, 34A34, 35G25, 35N10, 35Q53, 37K05, 37K10, 37K35, 58J70, 58J72.

Keywords: torus diffeomorphisms; vector fields; differential forms; versal deformation; central extension; LiePoisson structures; Casimir invariants; central extension.

doi:10.15330/ms.48.2.124-133

(C) D. Blackmore ${ }^{\mathrm{a}}$, A. Prykarpatski ${ }^{\mathrm{b}}$, M. Vovk ${ }^{\mathrm{c}}$, P. Pukach ${ }^{\mathrm{d}}$, Ya. Prykarpatsky ${ }^{\mathrm{e}}, 2017$ 
for an arbitrary constant vector $z \in \mathbb{T}^{n}$. Assuming that the vector-function $a \in$ $C^{1}\left(\mathbb{R} \times \mathbb{T}^{n} ; \mathbb{E}^{n}\right)$, it follows from the classical Cauchy theorem [11] on the existence and unicity of the solution to (3) and (4) that we can obtain a unique solution to the vector equation (3) as some function $\Phi \in C^{1}\left(\mathbb{R} \times \mathbb{T}^{n} ; \mathbb{T}^{n}\right), x=\Phi(t, z)$, such that the matrix $\partial \Phi(t, z) / \partial z$ is nondegenerate for all $t \in \mathbb{R}$ sufficiently close to $t^{(0)} \in \mathbb{R}$. Hence, the Implicit Function Theorem ([11,12]) implies that there exists a mapping $\Psi: \mathbb{R} \times \mathbb{T}^{n} \rightarrow \mathbb{T}^{n}$, such that

$$
\Psi(t, x)=z
$$

for every $z \in \mathbb{T}^{n}$ and all $t \in \mathbb{R}$ sufficiently enough to $t^{(0)} \in \mathbb{R}$. Supposing now that the functional vector $\Psi(t, x)=\left(\psi^{(1)}(t, x), \psi^{(2)}(t, x), \ldots, \psi^{(n)}(t, x)\right)^{\top},(t, x) \in \mathbb{R} \times \mathbb{T}^{n}$, is constructed, from the arbitrariness of the parameter $z \in \mathbb{T}^{n}$ one can deduce that all functions $\psi^{(j)}: \mathbb{R} \times$ $\mathbb{T}^{n} \rightarrow \mathbb{T}^{1}, j=\overline{1, n}$, are functionally independent invariants of the vector field equation (2), that is $A \psi^{(j)}=0, j=\overline{1, n}$. Thus, the vector field equation (2) has exactly $n \in \mathbb{Z}_{+}$functionally independent invariants, which make it possible, in particular, to solve the Cauchy problem posed above. Namely, let a mapping $\alpha: \mathbb{T}^{n} \rightarrow \mathbb{R}$ be chosen such that $\left.\alpha(\Psi(t, x))\right|_{t=t^{(0)}}=$ $\psi^{(0)}(x)$ for all $x \in \mathbb{T}^{n}$ and a fixed $t^{(0)} \in \mathbb{R}$. Inasmuch as the superposition of functions $\alpha \circ \Psi: \mathbb{R} \times \mathbb{T}^{n} \rightarrow \mathbb{T}^{1}$ is, evidently, also an invariant for the equation (2), it provides the solution to this Cauchy problem, which we can formulate as the following result.

Proposition 1. The linear equation (2), generated by the vector field (3) on the torus $\mathbb{T}^{n}$, has exactly $n \in \mathbb{Z}_{+}$functionally independent invariants.

Consider now a Plucker type ([16]) differential form $\chi^{(n)} \in \Lambda^{n}\left(\mathbb{T}^{n}\right)$ on the torus $\mathbb{T}^{n}$ as

$$
\chi^{(n)}:=d \psi^{(1)} \wedge d \psi^{(2)} \wedge \ldots \wedge d \psi^{(n)},
$$

generated by the vector $\Psi: \mathbb{R}^{n} \times \mathbb{T}^{n} \rightarrow \mathbb{T}^{n}$ of independent invariants (5), depending additionally on $n \in \mathbb{Z}_{+}$parameters $t \in \mathbb{R}^{n}$, where by definition, for any $k=\overline{1, n}$

$$
d \psi^{(k)}:=\left\langle\frac{\partial \psi^{(k)}}{\partial x}, d x\right\rangle+\sum_{j=1}^{n} \frac{\partial \psi^{(k)}}{\partial t_{j}} d t_{j}
$$

on the manifold $\mathbb{R}^{n} \times \mathbb{T}^{n}$. As follows from the Frobenius theorem $[11,13,16]$, the Plucker type differential form (6) is for all fixed parameters $t \in \mathbb{R}^{n}$ nonzero on the manifold $\mathbb{T}^{n}$ owing to the functional independence of the invariants (5). It is easy to see that at the fixed parameters $t \in \mathbb{R}^{n}$ the following [25] Jacobi-Mayer type relationship

$$
\begin{gathered}
\left|\frac{\partial \Psi}{\partial x}\right|^{-1} d \psi^{(1)} \wedge d \psi^{(2)} \wedge \ldots \wedge d \psi^{(n)}=\left(d x_{1}-\sum_{j=\overline{1, n}} a_{j}^{(1)}(t, x) d t_{j}\right) \wedge \\
\wedge\left(d x_{2}-\sum_{j=\overline{1, n}} a_{j}^{(2)}(t, x) d t_{j}\right) \wedge \ldots \wedge\left(d x_{n}-\sum_{j=\overline{1, n}} a_{j}^{(m)}(t, x) d t_{j}\right)
\end{gathered}
$$

holds for $k=\overline{1, n}$ on the manifold $\mathbb{R}^{n} \times \mathbb{T}^{n}$, where $\left|\frac{\partial \Psi}{\partial x}\right|$ is the determinant of the Jacobi mapping $\frac{\partial \Psi}{\partial x}: T\left(\mathbb{T}^{n}\right) \rightarrow T\left(\mathbb{T}^{n}\right)$ of the mapping (5) subject to the torus variables $x \in \mathbb{T}^{n}$. On the right-hand side of (8) one has the volume measure on the torus $\mathbb{T}^{n}$, which is naturally dependent on $t \in \mathbb{R}^{n}$ owing to the general vector field relationships (3). Taking into account that for all $k=\overline{1, n}$ the full differentials $d \psi^{(k)}=0$, that is vanishing on $\mathbb{R}^{n} \times \mathbb{T}^{n}$, the 
corresponding substitution of the reduced differentials $d \psi^{(k)} \in C^{2}\left(\mathbb{R}^{n} \times \mathbb{T}^{n} ; \Lambda^{1}\left(\mathbb{T}^{n}\right)\right), k=\overline{1, n}$, into (8) easily gives rise, in particular, to the following set of the compatible vector field relationships

$$
\frac{\partial \Psi}{\partial t_{s}}+\sum_{j, k=1, n}\left[\left(\frac{\partial \Psi}{\partial x}\right)_{j k}^{-1} \frac{\partial \psi^{(k)}}{\partial t_{s}}\right] \frac{\partial \Psi}{\partial x_{j}}=0,
$$

for all $s=\overline{1, n}$. The latter property, as it was demonstrated by M. G. Pfeiffer in [20-25], makes it possible to solve effectively the classical M. A. Buhl problem [8-10] and has interesting applications $[6,16]$ in the theory of completely integrable dynamical systems of heavenly type [15] and versally deformed operator structures [4] on toroidal manifilds.

1.1. Vector field hierarchies on the torus with "spectral" parameter and the LaxSato integrable heavenly dynamical systems. Consider some naturally ordered infinite set of parametric vector fields (1) on the infinite dimensional toroidal manifold $\mathbb{R}^{\mathbb{Z}_{+}} \times \mathbb{T}^{n}$ in the form

$$
A^{(k)}=\frac{\partial}{\partial t_{k}}+\left\langle a^{(k)}(t, x ; \lambda), \frac{\partial}{\partial x}\right\rangle+a_{0}^{(k)}(t, x ; \lambda) \frac{\partial}{\partial \lambda}:=\frac{\partial}{\partial t_{k}}+\mathrm{A}^{(k)},
$$

where $t_{k} \in \mathbb{R}, k \in \mathbb{Z}_{+},(t, x ; \lambda) \in\left(\mathbb{R}^{\mathbb{Z}_{+}} \times \mathbb{T}^{n}\right) \times \mathbb{C}$ are the evolution parameters, and the dependence of smooth vectors $\left(a_{0}^{(k)}, a^{(k)}\right)^{\top} \in \mathbb{E} \times \mathbb{E}^{n}, k \in \mathbb{Z}_{+}$, on the "spectral" parameter $\lambda \in \mathbb{C}$ is assumed to be holomorphic. Suppose now that the infinite hierarchy of linear equations

$$
A^{(k)} \psi=0
$$

for $k \in \mathbb{Z}_{+}$has exactly $n+1 \in \mathbb{Z}_{+}$common functionally independent invariants $\psi^{(j)}(\lambda) \in$ $C^{2}\left(\mathbb{R}^{\mathbb{Z}_{+}} \times \mathbb{T}^{n} ; \mathbb{C}\right), j=\overline{0, n}$ on the torus $\mathbb{T}^{n}$, suitably depending on the parameter $\lambda \in \mathbb{C}$. Then, owing to the existence theory $([11,12])$ for ordinary differential equations depending on the "spectral" parameter $\lambda \in \mathbb{C}$, these invariants may be assumed to be such that allow analytical continuation in the parameter $\lambda \in \mathbb{C}$ both inside the disc $\mathbb{D}_{+}^{1} \subset \mathbb{C}$ of some disc $\mathbb{D}^{1} \subset \mathbb{C}$ and subject to the parameter $\lambda^{-1} \in \mathbb{C},|\lambda| \rightarrow \infty$, outside $\mathbb{D}_{-}^{1} \subset \mathbb{C}$ of this disc $\mathbb{D}^{1} \subset \mathbb{C}$. This means that as $|\lambda| \rightarrow \infty$ we have the following expansions:

$$
\begin{gathered}
\psi^{(0)}(\lambda) \sim \lambda+\sum_{k=0}^{\infty} \psi_{k}^{(0)}(\tau, x) \lambda^{-k} \\
\psi^{(1)}(\lambda) \sim \sum_{k=0}^{\infty} \tau_{k}^{(1)}(t, x) \psi_{0}(\lambda)^{k}+\sum_{k=1}^{\infty} \psi_{k}^{(1)}(\tau, x) \psi_{0}(\lambda)^{-k}, \\
\psi^{(2)}(\lambda) \sim \sum_{k=0}^{\infty} \tau_{k}^{(2)}(t, x) \psi_{0}(\lambda)^{k}+\sum_{k=1}^{\infty} \psi_{k}^{(2)}(\tau, x) \psi_{0}(\lambda)^{-k}, \ldots, \\
\psi^{(n)}(\lambda) \sim \sum_{k=0}^{\infty} \tau_{k}^{(n)}(t, x) \psi_{0}(\lambda)^{k}+\sum_{k=1}^{\infty} \psi_{k}^{(n)}(\tau, x) \psi_{0}(\lambda)^{-k},
\end{gathered}
$$

where we took into account that $\psi^{(0)}(\lambda) \in C^{2}\left(\mathbb{R}^{\mathbb{Z}_{+}} \times \mathbb{T}^{n} ; \mathbb{C}\right), \lambda \in \mathbb{C}$, is the basic invariant solution to the equations (11), the functions $\tau \in C^{2}\left(\mathbb{R}^{\mathbb{Z}_{+}} \times \mathbb{T}^{n} ; \mathbb{R}^{n \times \mathbb{Z}_{+}}\right)$for all $s=\overline{1, n}, l \in \mathbb{Z}_{+}$, are assumed to be independent and $\psi_{k}^{(j)} \in C^{2}\left(\mathbb{R}^{\mathbb{Z}_{+}} \times \mathbb{T}^{n} ; \mathbb{R}\right)$ for all $k \in \mathbb{N}, j=\overline{0, n}$, are arbitrary invariants. Write down now the condition (8) on the manifold $\mathbb{C} \times \mathbb{T}^{n}$ in the form $\lambda \in \mathbb{C}$

$$
\left|\frac{\partial \Psi}{\partial \mathrm{x}}\right|^{-1} d \psi^{(0)} \wedge d \psi^{(1)} \wedge d \psi^{(2)} \wedge \ldots \wedge d \psi^{(n)}=\left(d \lambda-\sum_{j=\overline{1, n}} a_{j}^{(0)}(t, \mathrm{x}) d t_{j}\right) \wedge
$$




$$
\wedge\left(d x_{1}-\sum_{j=1, n} a_{j}^{(1)}(t, \mathrm{x}) d t_{j}\right) \wedge\left(d x_{2}-\sum_{j=1, n} a_{j}^{(2)}(t, \mathrm{x}) d t_{j}\right) \wedge \ldots \wedge\left(d x_{n}-\sum_{j=\overline{1, n}} a_{j}^{(m)}(t, \mathrm{x}) d t_{j}\right)
$$

where $\mathrm{x}:=(\lambda, x) \in \mathbb{C} \times \mathbb{T}^{n},\left|\frac{\partial \Psi}{\partial \mathrm{x}}\right|$ is the Jacobi determinant of the mapping $\Psi:=\left(\psi^{(0)}, \psi^{(1)}\right.$, $\left.\psi^{(2)}, \ldots, \psi^{(n)}\right)^{\top} \in C^{2}\left(\mathbb{C} \times\left(\mathbb{R}^{\mathbb{Z}_{+}} \times \mathbb{T}^{n}\right) ; \mathbb{C}^{n+1}\right)$ on the manifold $\mathbb{C} \times \mathbb{T}^{n}$. Inasmuch this mapping subject to the parameter $\lambda \in \mathbb{C}$ has analytical continuation [12] inside $\mathbb{D}_{+}^{1} \subset \mathbb{C}$ of the circle $\mathbb{D}^{1} \subset \mathbb{C}$ and subject to the parameter $\lambda^{-1} \in \mathbb{C}$ as $|\lambda| \rightarrow \infty$ outside $\mathbb{D}_{-}^{1} \subset \mathbb{C}$ of this disc $\mathbb{D}^{1} \subset \mathbb{C}$, one can easily obtain from the vanishing differential expressions

$$
d \psi^{(j)}=\left\langle\frac{\partial \psi^{(j)}}{\partial \mathrm{x}}, d \mathrm{x}\right\rangle+\sum_{k=0}^{\infty} \frac{\partial \psi^{(j)}}{\partial \tau_{k}^{(j)}} d \tau_{k}^{(j)}=0
$$

for all $j=\overline{0, n}$ and the relationship (13) on the extended manifold $\mathbb{C} \times \mathbb{T}^{n} \times \mathbb{T}^{\infty}$ of the independent variables $\mathrm{x} \in \mathbb{C} \times \mathbb{T}^{n}$, evolving analytically with respect to the parameters $\tau_{k}^{(j)} \in \mathbb{R}, j=\overline{1, n}, k \in \mathbb{Z}_{+}$, the following Lax-Sato criterion:

$$
\left(\left|\frac{\partial \Psi}{\partial \mathrm{x}}\right|^{-1} d \psi^{(0)} \wedge d \psi^{(1)} \wedge d \psi^{(2)} \wedge \ldots \wedge d \psi^{(n)}\right)_{-}=0
$$

where $(\ldots)_{-}$means the asymptotic part of an expression in the bracket, depending on the parameter $\lambda^{-1} \in \mathbb{C}$ as $|\lambda| \rightarrow \infty$. The substitution of expressions (14) into (15) easily yields

$$
-\frac{\partial \Psi}{\partial \tau_{k}^{(j)}}=\left[\left(\frac{\partial \Psi}{\partial \mathrm{x}}\right)_{0 j}^{-1} \psi^{(0)}(\lambda)^{k}\right]_{+} \frac{\partial \Psi}{\partial \lambda}+\sum_{s=1}^{n}\left[\left(\frac{\partial \Psi}{\partial \mathrm{x}}\right)_{s j}^{-1} \psi^{(0)}(\lambda)^{k}\right]_{+} \frac{\partial \Psi}{\partial x_{s}}
$$

for all $k \in \mathbb{Z}_{+}, j=\overline{1, n}$. These relationships (16) comprise an infinite hierarchy of Lax-Sato compatible $[29,30]$ linear equations, where $(\ldots)_{+}$denotes the asymptotic part of an expression in the bracket, depending on nonnegative powers of the complex parameter $\lambda \in \mathbb{C}$. As for the independent functional parameters $\tau_{k}^{(j)} \in C^{2}\left(\mathbb{R}^{\mathbb{Z}_{+}} \times \mathbb{T}^{n} ; \mathbb{R}\right)$ for all $k \in \mathbb{Z}_{+}, j=\overline{1, n}$, one can state their functional independence by taking into account their a priori linear dependence on the independent evolution parameters $t_{k} \in \mathbb{R}, k \in \mathbb{Z}_{+}$. On the other hand, taking into account the explicit form of the hierarchy of equations (16), following [6], it is not hard to show that the corresponding vector fields

$$
\mathrm{A}_{k}^{(j)}:=\left[\left(\frac{\partial \Psi}{\partial \mathrm{x}}\right)_{0 j}^{-1} \psi^{(0)}(\lambda)^{k}\right]_{+} \frac{\partial}{\partial \lambda}+\sum_{s=1}^{n}\left[\left(\frac{\partial \Psi}{\partial \mathrm{x}}\right)_{s j}^{-1} \psi^{(0)}(\lambda)^{k}\right]_{+} \frac{\partial}{\partial x_{s}}
$$

on the manifold $\mathbb{C} \times \mathbb{T}^{n}$ satisfy for all $k, m \in \mathbb{Z}_{+}, j, l=\overline{1, n}$, the Lax-Sato compatibility conditions

$$
\frac{\partial \mathrm{A}_{m}^{(l)}}{\partial \tau_{k}^{(j)}}-\frac{\partial \mathrm{A}_{k}^{(j)}}{\partial \tau_{m}^{(l)}}=\left[\mathrm{A}_{k}^{(j)}, \mathrm{A}_{m}^{(l)}\right],
$$

which are equivalent to the independence of the all functional parameters $\tau_{k}^{(j)} \in C^{1}\left(\mathbb{R}^{\mathbb{Z}_{+}} \times\right.$ $\left.\mathbb{T}^{n} ; \mathbb{R}\right), k \in \mathbb{Z}_{+}, j=\overline{1, n}$. As a corollary of the analysis above, one can show that the infinite hierarchy of vector fields (10) is a linear combination of the basic vector fields (17) and also satisfies the Lax type compatibility condition (18). Inasmuch the coefficients of vector fields (17) are suitably smooth functions on the manifold $\mathbb{R}^{\mathbb{Z}_{+}} \times \mathbb{T}^{n}$, the compatibility conditions 
(18) yield the corresponding sets of differential-algebraic relationships on their coefficients, which have the common infinite set of invariants, thereby comprising an infinite hierarchy of completely integrable so called heavenly nonlinear dynamical systems on the corresponding multidimensional functional manifolds. That is, all of the above can be considered as an introduction to a recently devised $[6,7,15,29,30]$ constructive algorithm for generating infinite hierarchies of commuting to each other vector fields on the toroidal manifolds $\mathbb{R}^{\mathbb{Z}_{+}} \times \mathbb{T}^{n}, n \in$ $\mathbb{Z}_{+}$, analytically depending on the complex parameter $\lambda \in \mathbb{C}$.

The important problem consisting in effective description of the corresponding integrable versal deformations $[2,3]$ of these infinite hierarchies of vector fields on the toroidal manifolds $\mathbb{R}^{\mathbb{Z}_{+}} \times \mathbb{T}^{n}, n \in \mathbb{Z}_{+}$, analytically depending on the complex parameter $\lambda \in \mathbb{C}$, is a main topic of next Sections following below.

\section{Versal deformations of vector fields and differential forms.}

2.1. Deformations. We consider a smooth vector field $A \in \Gamma\left(T\left(\mathbb{T}^{n}\right)\right)$ on the $n$-dimensional torus $\mathbb{T}^{n}$. A deformation of the vector field $A \in \Gamma\left(T\left(\mathbb{T}^{n}\right)\right)$ we will call a vector field $A(\tau) \in$ $\Gamma\left(T\left(\mathbb{T}^{n}\right)\right)$, which depends analytically on the parameter $\tau \in \mathbb{C}^{k}, k \in \mathbb{Z}_{+}$, in some vicinity of the point $\tau=0 \in \mathbb{C}^{k}$, and such that $A(0)=A$. The space of parameters $\Upsilon\left\{\tau \in \mathbb{C}^{k}\right\}$ is often called a base of the deformation. Similarly will consider a differential 1 -form $l \in \Lambda^{1}\left(\mathbb{T}^{n}\right)$ on the $n$-dimensional torus $\mathbb{T}^{n}$ and its related deformation $l(\tau) \in \Lambda^{1}\left(\mathbb{T}^{n}\right)$, which depends analytically on the parameter $\tau \in \mathbb{C}^{k}, k \in \mathbb{Z}_{+}$, in some vicinity of the point $\tau=0 \in \mathbb{C}^{k}$ and such that $l(0)=l$.

Definition 1. Two vector fields deformations $A(\tau)$ and $B(\tau) \in \Gamma\left(T\left(\mathbb{T}^{n}\right)\right)$ are called equivalent, if there exists such a deformation $g(\tau) \in \operatorname{Diff}\left(\mathbb{T}^{n}\right)$ of the identity $I d \in \operatorname{Diff}\left(\mathbb{T}^{n}\right)$, that $A d_{g(\tau)} A(\tau)=B(\tau)$, where $a d: \operatorname{Diff}\left(\mathbb{T}^{n}\right) \times \Gamma\left(T\left(\mathbb{T}^{n}\right)\right) \rightarrow \Gamma\left(T\left(\mathbb{T}^{n}\right)\right)$ is the usual $[2,3,5,14]$ adjoint mapping of the space $\operatorname{Diff}\left(\mathbb{T}^{n}\right)$ on $\Gamma\left(T\left(\mathbb{T}^{n}\right)\right)$. Similarly, two 1-form deformations $l(\tau)$ and $p(\tau) \in \Lambda^{1}\left(\mathbb{T}^{n}\right)$ are called equivalent, if there exists such a deformation $g(\tau) \in \operatorname{Diff}\left(\mathbb{T}^{n}\right)$ of the identity $I \in \operatorname{Diff}\left(\mathbb{T}^{n}\right)$, that $A d_{g(\tau)}^{*} l(\tau)=p(\tau)$, where $A d^{*}: \operatorname{Diff}\left(\mathbb{T}^{n}\right) \times \Lambda^{1}\left(\mathbb{T}^{n}\right) \rightarrow \Lambda^{1}\left(\mathbb{T}^{n}\right)$ is the usual adjoint mapping of the space Diff $\left(\mathbb{T}^{n}\right)$ on $\Lambda^{1}\left(\mathbb{T}^{n}\right)$.

Let $\varphi$ be a germ of a holomorphic at zero mapping $\mathbb{C}^{m} \rightarrow \mathbb{C}^{k}$, that is a set of converging at $0 \in \mathbb{C}^{m}$ degree series of complex variables, and assume that $\varphi(0)=0$. The mapping $\varphi: \Upsilon\left\{\sigma \in \mathbb{C}^{m}\right\} \rightarrow \Upsilon\left\{\tau \in \mathbb{C}^{m}\right\}$ defines evidently a new deformation $\check{\varphi} l(\sigma) \in \Lambda^{1}\left(\mathbb{T}^{n}\right)$ of the 1-form $l \in \Lambda^{1}\left(\mathbb{T}^{n}\right)$ and a new deformation $\hat{\varphi} A(\sigma)$ of the vector field $A \in \Gamma\left(T\left(\mathbb{T}^{n}\right)\right)$ via the expressions

$$
(\check{\varphi} l)(\sigma)=l(\varphi(\sigma)), \quad(\hat{\varphi} A)(\sigma)=A(\varphi(\sigma))
$$

on the deformation base $\Upsilon\left\{\sigma \in \mathbb{C}^{k}\right\}$.

Definition 2. The deformation $(\check{\varphi} l)(\sigma) \in \Lambda^{1}\left(\mathbb{T}^{n}\right)$ is called induced from the deformation $l(\tau) \in \Lambda^{1}\left(\mathbb{T}^{n}\right)$ under the mapping $\varphi: \Upsilon\left\{\sigma \in \mathbb{C}^{m}\right\} \rightarrow \Upsilon\left\{\tau \in \mathbb{C}^{m}\right\}$. Similarly, the deformation $(\hat{\varphi} A)(\sigma) \in \Gamma\left(T\left(\mathbb{T}^{n}\right)\right)$ is called induced from the deformation $A(\tau) \in \Gamma\left(T\left(\mathbb{T}^{n}\right)\right)$ under the mapping $\varphi: \Upsilon\left\{\sigma \in \mathbb{C}^{k}\right\} \rightarrow \Upsilon\left\{\tau \in \mathbb{C}^{m}\right\}$.

\subsection{Versal deformations.}

Definition 3. A vector field deformation $A(\tau) \in \Gamma\left(T\left(\mathbb{T}^{n}\right)\right)$ is called $[2,3,14]$ versal, if it generates every other deformation $B(\sigma) \in \Gamma\left(T\left(\mathbb{T}^{n}\right)\right)$ of the vector field $A \in \Gamma\left(T\left(\mathbb{T}^{n}\right)\right)$, that is there exists such a mapping $\varphi: \Upsilon\left\{\sigma \in \mathbb{C}^{k}\right\} \rightarrow \Upsilon\left\{\tau \in \mathbb{C}^{m}\right\}$ and a deformation 
$g(\tau) \in \operatorname{Diff}\left(\mathbb{T}^{n}\right)$ of the identity $I d \in \operatorname{Diff}\left(\mathbb{T}^{n}\right)$ that it is equivalent to the deformation obtained from the induced deformation $A(\varphi(\tau)) \in \Gamma\left(T\left(\mathbb{T}^{n}\right)\right)$

$$
B(\sigma)=A d_{g(\sigma)}(\hat{\varphi} A)(\sigma)
$$

on the deformation base $\Upsilon\left\{\sigma \in \mathbb{C}^{k}\right\}$. Similarly, a 1-form deformation $l(\tau) \in \Lambda^{1}\left(\mathbb{T}^{n}\right)$ is called versal, if it generates every other 1-form deformation $p(\tau) \in \Lambda^{1}\left(\mathbb{T}^{n}\right)$ of the 1-form $l \in \Lambda^{1}\left(\mathbb{T}^{n}\right)$, that is there exists such a mapping $\varphi: \Upsilon\left\{\sigma \in \mathbb{C}^{k}\right\} \rightarrow \Upsilon\left\{\tau \in \mathbb{C}^{m}\right\}$ and a deformation $g(\sigma) \in \operatorname{Diff}\left(\mathbb{T}^{n}\right)$ of the identity $I \in \operatorname{Diff}\left(\mathbb{T}^{n}\right)$ that it is equivalent to the deformation obtained from the induced deformation $p(\varphi(\sigma)) \in \Lambda^{1}\left(\mathbb{T}^{n}\right)$

$$
p(\sigma)=A d_{g(\sigma)}^{*}(\check{\varphi} l)(\sigma)
$$

on the deformation base $\Upsilon\left\{\sigma \in \mathbb{C}^{k}\right\}$.

\section{Versality and transversality.}

3.1. Transversality. Let $N \subset M$ be a smooth submanifold of a manifold $M$. Consider a smooth mapping $A: \Upsilon \rightarrow M$, and let a point $\tau \in \Upsilon$ for which $A(\tau) \in N$.

Definition 4. A mapping $A: \Upsilon \rightarrow M$ is called transvesal ( $[2,3])$ to the submanifold $N$ $\subset M$, if

$$
T_{A(\tau)}(M)=T_{A(\tau)}(N)+A_{*} T_{\tau}(\Upsilon) .
$$

As the diffeomorphism group Diff $\left(\mathbb{T}^{n}\right)$ naturally acts on a fixed vector field $A \in \Gamma\left(T\left(\mathbb{T}^{n}\right)\right)$, its orbit $\operatorname{Or}\left(A ; \operatorname{Diff}\left(\mathbb{T}^{n}\right)\right)=A d_{\operatorname{Diff}\left(\mathbb{T}^{n}\right)} A \subset \Gamma\left(T\left(\mathbb{T}^{n}\right)\right)$. Thus, a deformation $A(\tau) \in \Gamma\left(T\left(\mathbb{T}^{n}\right)\right)$ can be considered as a mapping $A: \Upsilon \rightarrow \Gamma\left(T\left(\mathbb{T}^{n}\right)\right)$ of the deformation base $\Upsilon\left\{\sigma \in \mathbb{C}^{m}\right\}$ into the space of vector fields $\Gamma\left(T\left(\mathbb{T}^{n}\right)\right)$ on the torus $\mathbb{T}^{n}$. The following lemma $([3,14])$ holds.

Lemma 1. A deformation $A(\tau) \in \Gamma\left(T\left(\mathbb{T}^{n}\right)\right)$ is versal iff the mapping $A:\left\{\tau \in \mathbb{C}^{m}\right\} \rightarrow$ $\Gamma\left(T\left(\mathbb{T}^{n}\right)\right)$ is transversal to the orbit of the corresponding element $A \in \Gamma\left(T\left(\mathbb{T}^{n}\right)\right)$, that is any deformation $B(\sigma)=A d_{g(\sigma)}^{*}(\hat{\varphi} A)(\sigma)$ on the deformation base $\Upsilon\left\{\sigma \in \mathbb{C}^{k}\right\}$ for some mapping $\varphi: \Upsilon\left\{\sigma \in \mathbb{C}^{k}\right\} \rightarrow \Upsilon\left\{\tau \in \mathbb{C}^{m}\right\}$.

Proof. Really, owing to the versality condition (20), for any deformation $B(\tau) \in \Gamma\left(T\left(\mathbb{T}^{n}\right)\right)$ of the vector field $A \in \Gamma\left(T\left(\mathbb{T}^{n}\right)\right)$ one has

$$
B(\tau)=A d_{g(\tau)}(\hat{\varphi} A)(\tau)
$$

on the deformation base $\Upsilon\left\{\tau \in \mathbb{C}^{m}\right\}$. Then, upon differentiating (23) with respect to $\tau \in \Upsilon$ one obtains that

$$
B_{*}(0) \xi=A_{*}(0) \hat{\varphi}(0) \xi+\left[C_{*}(0) \xi, A\right]
$$

for any $\xi \in T(\Upsilon)$, where $[\cdot, \cdot]$ is the usual commutator of vector fields on $\mathbb{T}^{n}$ and

$$
\left.\nabla_{\tau} \varphi(\tau)\right|_{\tau=0}:=\xi \in T_{0}(\Upsilon),\left.\nabla_{\tau} g(\tau)\right|_{\tau=0}:=C_{*}(0) \in \Gamma\left(T\left(\mathbb{T}^{n}\right)\right)
$$

Now it is easy to see that (24) is equivalent to the transversality condition (22), if to put $M:=\Gamma\left(T\left(\mathbb{T}^{n}\right)\right), N:=\operatorname{Or}\left(A ; \operatorname{Diff}\left(\mathbb{T}^{n}\right) \subset \Gamma\left(T\left(\mathbb{T}^{n}\right)\right)\right.$.

Consider now a smooth mapping $\alpha$ : $\operatorname{Diff}\left(\mathbb{T}^{n}\right) \rightarrow \Gamma\left(T\left(\mathbb{T}^{n}\right)\right)$, where

$$
\alpha(g):=A d_{g} A,
$$


which induces the tangent mapping $\alpha_{*}: \operatorname{diff}\left(\mathbb{T}^{n}\right) \rightarrow T_{A}\left(\Gamma\left(T\left(\mathbb{T}^{n}\right)\right)\right)$, where $\operatorname{diff}\left(\mathbb{T}^{n}\right):=$ $T_{I d}\left(\operatorname{Diff}\left(\mathbb{T}^{n}\right)\right.$ is the Lie algebra of vector fields on the torus $\mathbb{T}^{n}$ and acts as

$$
\alpha_{*} C=[C, A] .
$$

The kernel $K e r \alpha_{*}$ is a Lie subalgebra of vector fields commuting with the vector field $A \in$ $\Gamma\left(T\left(\mathbb{T}^{n}\right)\right)$ and is called its centralizer. It is also interesting to observe that the codimension $\operatorname{codim} \operatorname{Or}\left(A ; \operatorname{Diff}\left(\mathbb{T}^{n}\right)=\operatorname{dim} \operatorname{Ker} \alpha_{*}\right.$. As a result from reasoning in $[2,3,14]$ for small enough $\tau \in \Upsilon$ there exists an invertible mapping $\beta: V \times \Upsilon\left\{\tau \in \mathbb{C}^{m}\right\} \rightarrow \Gamma\left(T\left(\mathbb{T}^{n}\right)\right)$ for $V$ to be a submanifold of Diff( $\left(\mathbb{T}^{n}\right)$, transversal to the centralizer $\operatorname{dim} K e r \alpha_{*}$ and of maximal dimension $\operatorname{dim} V=\operatorname{dim} \operatorname{Or}\left(A ; \operatorname{Diff}\left(\mathbb{T}^{n}\right)\right.$, allowing the representation

$$
\beta(g, \tau)=A d_{g} A(\tau)
$$

on the deformation base $\Upsilon\left\{\tau \in \mathbb{C}^{m}\right\}$ for some $g \in V$. Let now $B(\sigma) \in \Gamma\left(T\left(\mathbb{T}^{n}\right)\right)$ be an arbitrary transversal deformation. Then it can be represented as $B(\sigma)=\beta(v, \tau)$, giving rise to the following expression

$$
B(\sigma)=A d_{g(\sigma)} A(\varphi(\sigma)),
$$

where $\varphi(\sigma):=\pi_{2} \beta^{-1}(B(\sigma)), g(\sigma):=\pi_{1} \beta^{-1}(B(\sigma))$ and $\pi_{1}$ and $\pi_{2}$ are projections of $V \times$ $\Upsilon\left\{\tau \in \mathbb{C}^{k}\right\}$ on the first and the second factor, respectively. The obtained expression (28) exactly means that this arbitrary deformation $B(\sigma) \in \Gamma\left(T\left(\mathbb{T}^{n}\right)\right)$ is versal, thus proving the lemma.

Consider now a 1-form deformation $l(\tau) \in \Lambda^{1}\left(\mathbb{T}^{n}\right)$ on the deformation base $\Upsilon\left\{\tau \in \mathbb{C}^{m}\right\}$. The same way as above one can prove the following dual to Lemma (1) proposition.

Proposition 2. A 1-form deformation $l(\tau) \in \Lambda^{1}\left(\mathbb{T}^{n}\right)$ is versal iff the mapping $l: \Upsilon\{\tau \in$ $\left.\mathbb{C}^{m}\right\} \rightarrow \Lambda^{1}\left(\mathbb{T}^{n}\right)$ is transversal to the orbit of the corresponding element $l \in \Lambda^{1}\left(\mathbb{T}^{n}\right)$, that is any deformation $p(\sigma)=A d_{g(\sigma)}^{*}(\check{\varphi} l)(\sigma)$ on the deformation base $\Upsilon\left\{\sigma \in \mathbb{C}^{k}\right\}$ for some mapping $\varphi: \Upsilon\left\{\sigma \in \mathbb{C}^{k}\right\} \rightarrow \Upsilon\left\{\tau \in \mathbb{C}^{m}\right\}$.

Being interested in describing versal deformations of pencils of differential forms, analytically depending on the "spectral" parameter $\lambda \in \mathbb{C}$, we will proceed below first to studying their orbits from the Marsden-Weinstein reduction theory point of view.

4. Torus diffeomorphism group and its orbits. Let us now consider the action of the diffeomorphism group Diff $\left(\mathbb{T}^{n}\right)$ on the space $\mathcal{G}:=\operatorname{diff}\left(\mathbb{T}^{n}\right) \ltimes \operatorname{diff}\left(\mathbb{T}^{n}\right)^{*}$, being the semidirect product $\Gamma\left(T\left(\mathbb{T}^{n}\right)\right) \ltimes \Lambda^{1}\left(\mathbb{T}^{n}\right) \simeq \operatorname{diff}\left(\mathbb{T}^{n}\right) \ltimes \operatorname{diff}\left(\mathbb{T}^{n}\right)^{*}$. It is well known $([18,19])$ that the semidirect sum $\mathcal{G}=\operatorname{diff}\left(\mathbb{T}^{n}\right) \ltimes \operatorname{diff}\left(\mathbb{T}^{n}\right)^{*}$ is a metrized Lie algebra with the Lie structure

$$
\left[a_{1} \ltimes l_{1}, a_{2} \ltimes l_{2}\right]:=\left[a_{1}, a_{2}\right] \ltimes\left(a d_{a_{1}}^{*} l_{2}-a d_{a_{2}}^{*} l_{1}\right),
$$

allowing to identify it with its adjoint space $\mathcal{G}^{*} \simeq \mathcal{G}$ via the nondegenerate and symmetric scalar product

$$
\left(a_{1} \ltimes l_{1}, a_{2} \ltimes l_{2}\right)=\left(l_{1}, a_{2}\right)+\left(l_{2}, a_{1}\right)
$$

for arbitrary $a_{1} \ltimes l_{1}, a_{2} \ltimes l_{2} \in \mathcal{G}^{*} \simeq \mathcal{G}$, where $(\cdot, \cdot): \Lambda^{1}\left(\mathbb{T}^{n}\right) \times \Gamma\left(T\left(\mathbb{T}^{n}\right)\right) \rightarrow \mathbb{C}$ is the standard pairing. 
Consider now the point product $\check{\mathcal{G}}:=\prod_{z \in \mathbb{S}^{1}} \tilde{\mathcal{G}}$ of Lie algebra $\mathcal{G}$ and endow it wit the central extension generated by a two-cocycle $\omega_{2}: \check{\mathcal{G}} \times \check{\mathcal{G}} \rightarrow \mathbb{C}$, where

$$
\omega_{2}\left(a_{1} \ltimes l_{1}, a_{2} \ltimes l_{2}\right):=\int_{\mathbb{S} 1}\left[\left(l_{1}, \partial a_{2} / \partial z\right)-\left(l_{2}, \partial a_{1} / \partial z\right)\right] d z
$$

for arbitrary $a_{1} \ltimes l_{1}, a_{2} \ltimes l_{2} \in \check{\mathcal{G}}$. Thus, the adjoint space $\check{\mathcal{G}}^{*}$ is a Poisson manifold $[2,5,28,31]$ endowed with the canonical Lie-Poisson structure

$$
\begin{gathered}
\{f, h\}_{0}:=(a \ltimes l,[\nabla f(a \ltimes l), \nabla h(a \ltimes l)])+ \\
+\int_{\mathbb{S}^{1}}\left[\left\langle\nabla f_{a}(a \ltimes l), \frac{\partial}{\partial z} \nabla h_{l}(a \ltimes l)\right\rangle-\left\langle\nabla h_{a}(a \ltimes l), \frac{\partial}{\partial z} \nabla f_{l}(a \ltimes l)\right\rangle\right] d z,
\end{gathered}
$$

where $f, h \in \mathcal{D}\left(\check{\mathcal{G}}^{*}\right), \nabla f(a \ltimes l):=\nabla f_{l}(a \ltimes l) \ltimes \nabla f_{a}(a \ltimes l) \in \check{\mathcal{G}}, \nabla h(a \ltimes l):=\nabla h_{l}(a \ltimes l) \ltimes$ $\nabla h_{a}(a \ltimes l) \in \check{\mathcal{G}}$ and $\nabla: \mathcal{D}\left(\check{\mathcal{G}}^{*}\right) \rightarrow \check{\mathcal{G}}$ is the usual functional gradient mapping. If to take now a constant vector field $d(a \ltimes l) / d s=J(\alpha):=\sum_{j, k=\overline{1, n}} \alpha_{j k} \partial / \partial x_{j} \ltimes d x_{k} \in \check{\mathcal{G}}^{*}$, depending on the constant parameters $\alpha_{k j} \in \mathbb{C}, j, k=\overline{1, n}$, one can construct $([17,27])$ by means of the Lie differentiation $L_{J(\alpha, \beta)}$ of the bracket (32) a new Poisson bracket

$$
\begin{gathered}
\left.\{f, h\}_{1}:=L_{J(\alpha, \beta)}\{f, h\}_{0}-\left\{L_{J(\alpha, \beta)} f, h\right\}_{0}-\left\{f, L_{J(\alpha, \beta}\right) h\right\}_{0}= \\
=(J(\alpha),[\nabla f(a \ltimes l), \nabla h(a \ltimes l)]),
\end{gathered}
$$

defined for any $f, h \in \mathcal{D}\left(\check{\mathcal{G}}^{*}\right)$ and satisfying the Jacobi condition.

Consider now the infinitesimal $\operatorname{Diff}\left(\mathbb{T}^{n}\right)$-actions on the space $\check{\mathcal{G}}^{*} \simeq \check{\mathcal{G}}$ subject to the Poisson brackets (32) and (33)

$$
d(a \ltimes l) / d \tau=\{h, a \ltimes l\}_{0}=\left(-\left[\nabla h_{l}, a\right]+\frac{\partial}{\partial z} \nabla h_{l}\right) \ltimes\left(a d_{\nabla h_{l}}^{*} l-a d_{a}^{*} \nabla h_{a}-\frac{\partial}{\partial z} \nabla h_{a}\right)
$$

subject to any function $h \in \mathcal{D}\left(\check{\mathcal{G}}^{*}\right)$ and

$$
d(a \ltimes l) / d \xi=\{f, a \ltimes l\}_{1}=-\sum_{j=\overline{1, n}} \alpha_{j k}\left[\nabla f_{l}, \partial / \partial x_{j}\right] \ltimes a d_{\nabla f_{l}}^{*} d x_{k}
$$

subject to a Casimir function $f \in \mathcal{D}\left(\check{\mathcal{G}}^{*}\right)$, respectively to the evolution parameters $\tau$ and $\xi \in \mathbb{C}$. Making use of the vector fields (34) and (35), one can construct the following integrable on the space $\check{\mathcal{G}}^{*}$ distributions:

$$
\mathcal{D}_{0}=\left\{\left(-\left[\nabla h_{l}, a\right]+\frac{\partial}{\partial z} \nabla h_{l}\right) \ltimes\left(a d_{\nabla h_{l}}^{*} l-a d_{a}^{*} \nabla h_{a}-\frac{\partial}{\partial z} \nabla h_{a}\right): h \in I_{1}\left(\check{\mathcal{G}}^{*}\right)\right\},
$$

where $I_{1}\left(\check{\mathcal{G}}^{*}\right)$ is the space of Casimir functions for the Poisson bracket (33), and

$$
\mathcal{D}_{1}=\left\{-\sum_{j, k=\overline{1, n}} \alpha_{j k}\left[\nabla f_{l}, \partial / \partial x_{j}\right] \ltimes a d_{\nabla f_{l}}^{*} d x_{k}: f \in \mathcal{D}\left(\check{\mathcal{G}}^{*}\right)\right\},
$$

as $\left[\mathcal{D}_{0}, \mathcal{D}_{0}\right] \subset \mathcal{D}_{0}$ and $\left[\mathcal{D}_{1}, \mathcal{D}_{1}\right] \subset \mathcal{D}_{1}$. The set of maximal integral submanifolds of (37) generates the foliation $\breve{\mathcal{G}}_{J}^{*} \backslash \mathcal{D}_{0}$, whose leaves are the intersections of fixed integral submanifolds $\check{\mathcal{G}}_{J}^{*} \subset \check{\mathcal{G}}^{*}$ of the distribution $\mathcal{D}_{1}$ passing through an element $a \ltimes l \in \mathcal{G}^{*}$ with the leaves of the distribution $\mathcal{D}_{0}$, generating a compatible system of the Pfeiffer-Lax-Sato type [15] vector field equations. If the foliation $\check{\mathcal{G}}_{J}^{*} \backslash \mathcal{D}_{0}$ is sufficiently smooth, one can define the quotient manifold $\check{\mathcal{G}}_{\text {red }}^{*}:=\check{\mathcal{G}}_{J}^{*} /\left(\check{\mathcal{G}}_{J}^{*} \backslash \mathcal{D}_{0}\right)$ with its associated projection mapping $\check{\mathcal{G}}_{J}^{*} \rightarrow \check{\mathcal{G}}_{\text {red }}^{*}$. The structure of the reduced manifold $\check{\mathcal{G}}_{\text {red }}^{*}$ is characterized by the following theorem. 
Theorem 1. On the manifold $\check{\mathcal{G}}_{\text {red }}^{*}$ the pair of Poisson structures $\{\cdot, \cdot\}_{0}$ and $\{\cdot, \cdot\}_{1}$ are compatible, that is for any parameter $\lambda \in \mathbb{R}$ the algebraic sum $\{\cdot, \cdot\}_{0}+\lambda\{\cdot, \cdot\}_{1}$ is Poisson too.

A proof of Theorem 1 is strongly based on the classical differential-geometric MarsdenWeinstein reduced space construction.

As a consequence of Theorem 1 and reasonings, based on the structure of the distribution (36), one can describe its invariants on and a leave $\check{\mathcal{G}}_{J}^{*}$ and present the related coordinates on the reduced manifold $\check{\mathcal{G}}_{\text {red }}^{*}=\check{\mathcal{G}}_{J}^{*} /\left(\check{\mathcal{G}}_{J}^{*} \backslash \mathcal{D}_{0}\right)$. Thus, the related with (34) reduced flow on the manifold $\check{\mathcal{G}}_{\text {red }}^{*}$ will present the canonical representation of the studied versal deformation subject to a metric Lie algebra generated by the semidirect $\operatorname{sum} \Gamma\left(T\left(\mathbb{T}^{n}\right)\right) \ltimes \Lambda^{1}\left(\mathbb{T}^{n}\right) \simeq$ $\operatorname{diff}\left(\mathbb{T}^{n}\right) \ltimes \operatorname{diff}\left(\mathbb{T}^{n}\right)^{*}$ of the smooth affine vector fields $\Gamma\left(T\left(\mathbb{T}^{n}\right)\right)$ on the torus $\mathbb{T}^{n}$ and its adjoint space $\Lambda^{1}\left(\mathbb{T}^{n}\right)$. As an example, for the one-dimensional torus $\mathbb{T}^{1} \simeq \mathbb{S}^{1}$ one can take a seed element $a \ltimes l:=v \partial / \partial x \ltimes u d x \in \check{\mathcal{G}}^{*}=\Gamma\left(T\left(\mathbb{S}^{1}\right)\right) \ltimes \Lambda^{1}\left(\mathbb{S}^{1}\right), x \in \mathbb{S}^{1}$, where $u, v \in C^{2}\left(\mathbb{S}^{1} \times \mathbb{S}^{1} ; \mathbb{R}\right)$ are some smooth functions. If the constant element $J(\alpha):=\alpha \partial / \partial x \ltimes d x \in \check{\mathcal{G}}^{*} \simeq \breve{\mathcal{G}}$, the space $I_{2}\left(\check{\mathcal{G}}^{*}\right)$ of the corresponding Casimir gradient easily ensue from (36), generating an interesting for applications compatible system of the Pfeiffer-Lax-Sato type vector field equations. Their detailed analytical structure is under preparation and will be presented in other place.

5. Acknowledgements. The authors cordially thank Prof. M. Błaszak, Prof. J. Cieślinski and Prof. B. Szablikowski for useful discussions of the results during the International Conference in Functional Analysis dedicated to the $125^{\text {th }}$ anniversary of Stefan Banach held on 18-23 September, 2017 in Lviv, Ukraine.

\section{REFERENCES}

1. R. Abraham, J. Marsden, Foundations of Mechanics, Second Edition, NY, Benjamin Cummings, 1978.

2. V.I. Arnold, Mathematical methods of classical mechanics, Springer, 1989.

3. V.I. Arnold, On matrices depending on parameters, Russian Math. Surveys, 26 (1971), 29-43.

4. D. Blackmore, A.K. Prykarpatsky, Versal deformations of a Dirac type differential operator, Journal of Nonlinear Mathematical Physics, 6 (1999), №3, 246-254.

5. D. Blackmore, A.K. Prykarpatsky, V.H. Samoylenko, Nonlinear dynamical systems of mathematical physics, World Scientific Publisher, NJ, USA, 2011.

6. L.V. Bogdanov, V.S. Dryuma, S.V. Manakov, Dunajski generalization of the second heavenly equation: dressing method and the hierarchy, J. Phys. A: Math. Theor., 40 (2007), 14383-14393.

7. L.V. Bogdanov, M.V. Pavlov, Linearly degenerate hierarchies of quasiclassical SDYM type, arXiv:1603.00238v2 [nlin.SI], 2016.

8. M.A. Buhl, Surles operateurs differentieles permutables ou non, Bull. des Sc. Math., 1928, S.2, t. LII, 353-361.

9. M.A. Buhl, Apercus modernes sur la theorie des groupes continue et finis, Mem. des Sc. Math., fasc. XXXIII, Paris, 1928.

10. M.A. Buhl, Apercus modernes sur la theorie des groupes continue et finis, Mem. des Sc. Math., fasc. XXXIII, Paris, 1928.

11. A. Cartan, Differential Forms, Dover Publisher, USA, 1971.

12. Earl A. Coddington, Norman Levinson, Theory of ordinary differential equations, International series in pure and applied mathematics, McGraw-Hill, 1955.

13. C. Godbillon, Geometrie Differentielle et Mecanique Analytique, Hermann Publ., Paris, 1969.

14. A. Edelman, E. Elmrothy, B. Kagstrom, A Geometric Approach to Perturbation Theory of Matrices and Matrix Pencils, Part I: Versal Deformations, March 23, 1995, Preprint: UMINF-95.09. 
15. O.E. Hentosh, Ya.A. Prykarpatsky, D. Blackmore, A.K. Prykarpatski, Lie-algebraic structure of LaxSato integrable heavenly equations and the Lagrange-d'Alembert principle, Journal of Geometry and Physics, 120 (2017), 208-227.

16. B.G. Konopelchenko, Grassmanians $\operatorname{Gr}(N-1, N+1)$, closed differential $N-1$ forms and $N$-dimensional integrable systems, arXiv:1208.6129v2 [nlin.SI], 2013.

17. F. Magri, Acta Applicandea Mathematica, 41 (1995), 247-270.

18. V. Ovsienko, Bi-Hamilton nature of the equation $u_{t x}=u_{x y} u_{y}-u_{y y} u_{x}$, arXiv:0802.1818v1, 2008.

19. V. Ovsienko, C. Roger, Looped cotangent virasoro algebra and non-linear integrable systems in dimension $2+1$, Commun. Math. Phys., 273 (2007), 357-378.

20. G. Pfeiffer, Generalisation de la methode de Jacobi pour l'integration des systems complets des equations lineaires et homogenes, Comptes Rendues de l'Academie des Sciences de l'URSS, 190 (1930), 405-409.

21. M.G. Pfeiffer, Sur la operateurs d'un systeme complet d'equations lineaires et homogenes aux derivees partielles du premier ordre d'une fonction inconnue, Comptes Rendues de l'Academie des Sciences de l'URSS, 190 (1930), 909-911.

22. M.G. Pfeiffer, La generalization de methode de Jacobi-Mayer, Comptes Rendues de l'Academie des Sciences de l'URSS, 191 (1930), 1107-1109.

23. M.G. Pfeiffer, Sur la permutation des solutions s'une equation lineaire aux derivees partielles du premier ordre, Bull. des Sc. Math., LII (1928), S.2, 353-361.

24. M.G. Pfeiffer, Quelgues additions au probleme de M. Buhl, Atti dei Congresso Internationale dei Matematici, Bologna, III (1928), 45-46.

25. M.G. Pfeiffer, La construction des operateurs d'une equation lineaire, homogene aux derivees partielles premier ordre, Journal du Cycle Mathematique, Academie des Sciences d'Ukraine, Kyiv, 1 (1931), 37-72. (in Ukrainian)

26. C. Popovici, Sur les fonctions adjointes de M. Buhl, Comptes Rendus, 145 (1907), 749.

27. A. Prykarpatsky, D. Blackmore, Versal deformations of a Dirac type differential operator, Journal of Nonlinear Mathematical Physics, 6 (1999), №3, 246-254.

28. A.G. Reyman, M.A. Semenov-Tian-Shansky, Integrable systems, The Computer Research Institute Publ., Moscow-Izhvek, 2003. (in Russian)

29. K. Takasaki, T. Takebe, SDiff(2) Toda equation - Hierarchy, Tau function, and symmetries, Letters in Mathematical Physics, 23 (1991) №2, 205-214.

30. K. Takasaki, T. Takebe, Integrable Hierarchies and dispersionless limit, Reviews in Mathematical Physics, 7 (1995), №05, 743-808.

31. L.A. Takhtadjian, L.D. Faddeev, Hamiltonian approach in soliton theory, Springer, Berlin-Heidelberg, 1987.

\footnotetext{
a Department of Mathematical Sciences at NJIT

University Heights, Newark, NJ, USA

denblac@gmail.com

b The Department of Physics, Mathematics and Computer Science of the Cracov University of Technology, Krakow, Poland

pryk.anat@cybergal.com

c,d Fundamental Sciences Institute

Lviv Polytechnical Universtity, Lviv, Ukraine

mira.vovk1@gmail.com

e The Department of Applied Mathematics

the University of Agriculture in Krakow, Poland

pryk.anat@cybergal.com
} 